\title{
CO-St (COVID 19- study): impact of the management of men versus women in the treatment of Covid 19. A Multi-Cen- tric Observational study
}

\section{Running title: COVID-19 Patients and Gender Differences}

\author{
Tiziana Ciarambino ${ }^{1}$, Filomena Pietrantonio ${ }^{2}$, Sara Rotunno ${ }^{3}$, Alessandra Fiorentini ${ }^{4}$, Rosalba Cipriani ${ }^{5}$, Giuseppe \\ Campagna $^{6}$, Giuseppe Straface ${ }^{7}$, Eleonora Pistella ${ }^{8}$, Mauro Giordano $^{9}$, Francesco Rosiello ${ }^{2,10 *}$, Orazio Valerio \\ Giannico $^{11}$, Federica Lorenzi ${ }^{12}$
}

\author{
${ }^{1}$ Internal Medicine Department, Hospital of Marcianise, Caserta, Italy \\ 2Internal Medicine Department, Hospital Castelli ASL Roma 6, Ariccia, Italy \\ 3Internal Medicine Department, Hospital of S Pietro Fatebenefratelli, Roma, Italy \\ 4Internal Medicine Department, Hospital Belcolle, Viterbo, Italy \\ 5Internal Medicine Department, Hospital F Spaziani, Frosinone, Italy \\ 6Internal Medicine Department, Hospital S. Maria Goretti, Latina, Italy \\ 7 Science Medical Department, AUSL Latina, Italy \\ 8 Internal Medicine Department, Hospital Vannini, Roma, Italy \\ 9Internal Medicine Department, University of Campania, Caserta, Italy \\ 10Dept. Public Health and Infectious Disease, Sapienza-University of Rome, Rome, Italy \\ 11Department of Prevention, Local Health Authority of Taranto, Taranto, Italy \\ 12Internal Medicine Department, Hospital L. Parodi Delfino, Colleferro, Italy \\ * Correspondence: francesco.rosiello@uniroma1.it
}

\begin{abstract}
:
Background: from December 2019 and the spreading of syndemics, a lot of medical centers registered data about their patients. In Italy, the most relevant quantity of patients was hospitalized in Internal Medicine wards. Methods: In this observational, retrospective cross-sectional study, all data of the COVID-19 patients, admitted Lazio hospitals, from March 01 to December 31, 2020, were collected and their Epidemiological data, demographics, signs and symptoms on admission, comorbidities, laboratory findings, chest radiography and CT findings, treatment received and mortality rate were analyzed by gender to find any differences of gravity of disease. Clinician details were registered on database (one for every hospital). Cost analysis was performed by length of stay and antiviral drugs use, using point of view of Italian Healthcare System. Results: 2256 patients with mean age of $71.01 \pm 28.02$ years were included. For men, frequency of hypertension, Chronic Ostructive Pulmonary Disease (COPD, use of oxygen therapy, Tocilizumab were significantly higher and epidemiological link was related to rehabilitation ward and community. The gender difference about hospitalization was one day more for man. No strong significant difference by gender in the death rate was observed. Considering antiviral drugs and hospitalization, a man costs $€ 591,2$ more than woman. Conclusions: In male patients, hypertension and COPD were observed more frequently and the epidemiological link was related to rehabilitation ward and community. In female subjects, the epidemiological link was related to Hospital and we observed significantly higher atypical chest- $X$ ray. Tocilizumab, oxygen therapy and antiviral drugs were prescribed more in male subjects. No differences by gender we report in other treatments and outcomes. Future studies should be analyzed to get a more comprehensive understanding of COVID-19 by gender.
\end{abstract}

Keywords: SARS-CoV-2; COVID-19; gender role; sex differences 


\section{Introduction}

A new Coronavirus in late 2019 resulted in several pneumonia cases in China, in the Hubei region (1). This virus quickly spreads to numerous other countries, and in February 2020, WHO defines the disease, triggered by this Coronavirus, as Covid-19 (2, 3). On 11 March 2020, WHO declares the world pandemic, with 118.000 cases in 114 countries and 4.291 deaths from COVID-19. The analysis by gender has reported a similar number of men and women affected by SARS-CoV-2 infection, although mortality seems to be higher in men. In this regard, it has been reported that the male gender is also more sensitive due to the higher number of smokers in men than in women. Different studies reported that male seem to be more susceptible to COVID-19-related complications, and they represent between $50 \%$ and $82 \%$ of the hospitalized patients (4-8). Different factors can contribute to COVID-19 response related to gender differences; it has been reported that the age is a factor that induce the modification of immune response. In fact, with aging, there is an aberrant chronic low-grade pro-inflammatory state, which may occur to a greater extent in females than in males (9). To this regard, immune senescence is a major contributory factor in the increased susceptibility of older adults to infection (10). Men experience a stronger 'inflammation' syndrome than women. In terms of COVID-19, it is interesting that the aging lung is characterized by a state of heightened basal inflammation, with levels of IL-6, amongst other cytokines, significantly higher in the BALF of healthy older adults when compared to their younger counterparts (11). The aim of this report is to analyze, the epidemiologic features, epidemiological link, comorbidity, drugs therapy and computerized tomography (CT) findings by gender in patients COVID-19 admitted to the different hospital in Lazio, Italy, from March 01 to December 31, 2020.

\section{Materials and Methods}

Sample size calculation: all patients admitted to participating centers were included in the study. According to the protocol approved by the Ethics Committee, the minimum number of patients per center is 250 .

Study design

In this multi-center retrospective cross sectional study, we included data of the COVID19 patients. Written informed consent was obtained from the patients for the publication of individual data from before enrolment. Different authors were responsible for data gathering. If data were missing from the records or clarification was needed, data were obtained by direct communication with attending doctors and other healthcare providers. A sensitivity analysis carried out to determine the robustness of the study and identify anomalous data.

Lazio 2 Ethical Committee approved this study as "55.21 Study", protocol 78111 04/05/2021.

Study setting and Population

We included data of the all COVID-19 patients ( $\mathrm{n}=2256)$, admitted to Internal Medicine wards of all different COVID-19 Hospital, of Lazio, Italy, from March 01 to December 31, 2020, collected from an electronic archive with laboratory confirmation of SARS-CoV-2 infection done at COVID-Hospital, Lazio using Real-time RT-PCR. We excluded all patients with negative laboratory tests for SARS-CoV-2 infection. According to the Italian Government protocol, patients were admitted centrally to the COVID-Hospitals from all cities of Lazio, without selectivity. After the admission in Internal Medicine ward, the sample was stratified by gender. Epidemiological data, demographics, signs and symptoms on admission, comorbidities, laboratory findings, chest radiography and CT 
findings, treatment received and mortality rate were analyzed by gender. Epidemiologic, demographic, clinical, laboratory, and management (multidisciplinary assessment, delivery methods, and treatments) data from patients' medical records of different COVID-Hospitals, Lazio, Italy, were collected using a predesign checklist including all patients from available clinical data record by 9 Lazio Hospitals.

Study Protocol

2256 patients with mean age of $71.01 \pm 28.02$ years were included in this study (56\% males).

The incubation period was defined as the interval between the potential earliest date of contact of the transmission source (wildlife or person with suspected or confirmed case) and the potential earliest date of symptom onset (i.e., cough, fever, fatigue, or myalgia). Laboratory confirmation of SARS-CoV-2 infection was done at COVID-Hospital, Lazio using Real-time RT-PCR (9). Other respiratory viruses, including influenza A virus, influenza B virus, respiratory syncytial virus, parainfluenza virus, and adenovirus were also tested for by real-time RT-PCR. Throat swab specimens from the upper respiratory tract that were obtained from all patients at admission, were maintained in viral transport medium. Sputum or endotracheal aspirates were obtained at admission for the identification of possible causative bacteria or fungi. Clinical outcomes were followed up to December 31, 2020. The patient had been discharged from the hospital per the following discharge criteria: body temperature returned to normal for more than 3 days; respiratory symptoms improved significantly; pulmonary imaging showed a significant improvement in acute exudative lesions; and nucleic acid test of respiratory specimens such as results of sputum and nasopharyngeal swabs were negative twice in a row (sampling interval $\geq 24 \mathrm{~h}$ ).

Measures and Data Analysis

Statistical analysis was performed using R 4.0.0 (released on 2020-04-24). Statistical significance was fixed to 0.05 . Categorical variables are reported as absolute and relative frequencies and compared through Chi Square test. Continuous variables are reported as mean \pm standard deviation (SD) and compared through Welch t-test. Missing data were excluded from analysis.

Cost Analysis

Cost analysis was performed using as proxy the hospitalization costs and use of antiviral drugs. Point of view of Italian national healthcare system was adopted.

\section{Results}

Baseline and clinical characteristics

2256 patients with mean age of $71.01 \pm 28.02$ years were included in this study (56\% males). $1405(62.27 \%)$ patients were admitted to COVID-Hospital for fever, 425 (18.8\%) for dyspnea and 426 (18.8\%) for cough.

The average incubation period was $7.5 \pm 1.0$ (range 6-9) days. The mean age in females and males were $70.01 \pm 25.01$ and $71.02 \pm 22.3$ years respectively $(p=0.313)$. Hypertension (51\% vs $48 \%$; $\mathrm{p}<0.001)$ and COPD (48\% vs $43 \%$; $\mathrm{p}<0.001)$ were common in male patients. In the male subjects the epidemiological link was related to rehabilitation (80\% vs 59\%; $\mathrm{p}<0.001)$ and community $(14 \%$ vs $4 \%$; $<<0.001)$; while in female it was related to hospital contagious ( $36 \%$ vs $5 \%$; $\mathrm{p}<0.001$ ). 
Table 1.

\begin{tabular}{|c|c|c|c|c|c|}
\hline Variable & Total $(n=2256)$ & Male $(n=1265)$ & $\begin{array}{c}\text { Female }(n= \\
991)\end{array}$ & $\mathbf{P}$ & Total \\
\hline \multicolumn{6}{|l|}{ Age (year) } \\
\hline Mean \pm SD & $71.01 \pm 28.02$ & $70.02 \pm 25.01$ & $\begin{array}{l}71.01 \pm \\
22.02\end{array}$ & 0.313 & 71. \\
\hline \multicolumn{6}{|l|}{ Comorbidity } \\
\hline COPD (\%) & $1037(45.96)$ & 607 (47.98) & 430 (43.39) & $<0.001$ & 103 \\
\hline $\begin{array}{c}\text { Hypertension } \\
(\%)\end{array}$ & 1127 (49.95) & $650(51.38)$ & 477 (48.13) & & 112 \\
\hline $\begin{array}{c}\text { Other (\%) } \\
\text { Incubation } \\
\text { period (days) }\end{array}$ & $92(4.07)$ & $8(0.63)$ & $84(8.47)$ & & \\
\hline Mean \pm SD & $7.50 \pm 1.90$ & $6.3 \pm 2.10$ & $9.2 \pm 1.10$ & $<0.001$ & 7. \\
\hline \multicolumn{6}{|l|}{$\begin{array}{c}\text { Presenting vital } \\
\text { sign }\end{array}$} \\
\hline SBP (mmHg) & $130.01 \pm 5.10$ & $125.01 \pm 3.00$ & $130.01 \pm 1.01$ & $<0.001$ & 130 \\
\hline DBP (mmHg) & $75.01 \pm 5.00$ & $75.01 \pm 3.01$ & $75.01 \pm 2.01$ & $>0.999$ & 75 \\
\hline RR (/minute) & $18.01 \pm 3.01$ & $15.01 \pm 2.02$ & $20.00 \pm 1.01$ & $<0.001$ & \\
\hline PR (/minute) & $60.01 \pm 2.00$ & $60.01 \pm 3.01$ & $60.00 \pm 4.00$ & $>0.999$ & 60 \\
\hline $\begin{array}{c}\text { Temperature } \\
\left({ }^{\circ} \mathrm{C}\right)\end{array}$ & $37.70 \pm 4.01$ & $38.01 \pm 2.00$ & $37.51 \pm 1.01$ & $<0.001$ & 37 \\
\hline \multicolumn{6}{|l|}{$\begin{array}{c}\text { Epidemiological } \\
\text { links }\end{array}$} \\
\hline $\begin{array}{c}\text { Rehabilitation } \\
(\%)\end{array}$ & 1604 (71.09) & 1015 (80.23) & $589(59.43)$ & $<0.001$ & 160 \\
\hline Community (\%) & 225 (9.97) & $180(14.22)$ & $45(4.54)$ & & \\
\hline Hospital (\%) & $427(18.92)$ & $70(5.53)$ & $357(36.02)$ & & 42 \\
\hline
\end{tabular}

${ }^{1}$ Baseline and clinical characteristics of studied population.

Imaging findings

According to chest CT scan findings, $86 \%$ of patients had bilateral pneumonia and the remaining $14.02 \%$ had unilateral pneumonia. As showed in Table 2, atypical chest- $X$ ray was more frequent in female $(41 \%$ vs $16 \%$; p <0.001), compare to male subjects, while abnormal Chest CT scan was significantly frequent in male $(84 \%$ vs $59 \%, \mathrm{p}<0.001$, respectively). As reported in Table 2, we report abnormalities distribution on the right and left lung, with bilateral involvement in female $(\mathrm{p}<0.001)$, compared to male subjects.

Table 2. Differences by gender regarding therapeutic measures, as well as imaging and laboratory findings.

\begin{tabular}{ccccc}
\hline Variables & Male (n = 1265) & Female (n= 991) & Total $(\mathbf{n}=\mathbf{2 2 5 6})$ & P \\
\hline $\begin{array}{c}\text { Therapeutic } \\
\text { measures }\end{array}$ & & & & \\
TCZ & $293(23.16)$ & $22(2.21)$ & $315(13.96)$ & $<0.001$ \\
Oxygen therapy & $902(71.30)$ & $609(61.45)$ & $1511(66.97)$ & \\
$\quad \begin{array}{l}\text { Other } \\
\text { Outcomes }\end{array}$ & $70(5.53)$ & $360(36.32)$ & $430(19.06)$ & $<0.001$ \\
$\begin{array}{c}\text { Need for intubation } \\
\text { Need for ICU }\end{array}$ & $199(15.73)$ & $210(21.19)$ & $409(18.12)$ & $<0.001$ \\
$\begin{array}{c}\text { admission } \\
\text { Mortality }\end{array}$ & $215(16.99)$ & $206(20.78)$ & & $<0.001$ \\
$\begin{array}{c}\text { Need for antiviral } \\
\text { agent }\end{array}$ & $100(7.90)$ & $102(10.29)$ & & \\
$\begin{array}{c}\text { Chest X ray } \\
\text { findings } \\
\text { Atypical }\end{array}$ & $751(59.36)$ & $473(47.72)$ & & $<0.001$ \\
\hline
\end{tabular}




\begin{tabular}{|c|c|c|c|}
\hline Typical & $1062(83.95)$ & 585 (59.03) & $<0.001$ \\
\hline \multicolumn{4}{|l|}{ Chest CT scan } \\
\hline Normal & $203(16.04)$ & $406(40.96)$ & $<0.001$ \\
\hline Abnormal & 1062 (83.95) & 585 (59.03) & \\
\hline $\begin{array}{l}\text { Distribution of } \\
\text { abnormalities on } \\
\text { chest CT scan }\end{array}$ & & & $<0.001$ \\
\hline Right lung & $300(23.71)$ & $310(31.28)$ & $<0.001$ \\
\hline Left lung & $302(23.87)$ & 307 (30.97) & \\
\hline
\end{tabular}

${ }^{1}$ CT: computed tomography; TCZ: Tocilizumab; CRP: C-Reactive protein, ESR: erythrocyte sedimentation rate.

While, in male we observe abnormalities distribution on the upper, lower and middle lobe $(p<0.001)$, compared to female subjects. We do not observe gender differences in relation to type of distribution of abnormalities on chest CT scan and in relation to type of abnormalities on chest CT scan $(\mathrm{p}=0.190)$.

Treatment

All patients received antiviral treatment. In male subjects we detected a higher use of the antiviral drugs (59\% vs $47 \%$, p $<0.001$, respectively), compared to female subjects. Male subjects had more need for oxygen therapy (71\% vs $61 \% ; \mathrm{p}<0.001)$. However, tocilizumab was prescribed more in male subjects $(23 \%$ vs $2 \%$; $<<0.001)$. No gender differences were observed in other treatment.

\section{Outcomes}

At the end of follow-up (December 31, 2020), 9\% of patients died. No strong significant difference by gender in the death rate was observed. As reported in Table 2, the significantly gender differences on the need for intubation $(21 \%$ in female compared to $16 \%$ in male, $\mathrm{p}<0.001)$; and admission in the intensive unit care $(21 \%$ in female compared to $17 \%$ in male, $\mathrm{p}<0.001$ ) were observed.

Cost analysis

In Italy, every day of hospitalization in internal medicine ward cost $€ 546,00$ (12). About Tocilizumab $10 \mathrm{ml}$, National Drugs Agency (AIFA) allows it only to patient that have all inclusion criteria and no exclusion ones. Inclusion criteria: a COVID-19 infection confirmed by microbiological test AND being treated with dexamethasone (or equivalent) AND have CRP> 75mg/L AND SaO2 <92\% in ambient air OR need for O2 supplementation AND not treated with an IL-6 inhibitor for COVID-19 during hospitalization and within 24-48 hours of starting respiratory support with high-flow nasal oxygen, continuous positive airway pressure (CPAP), or mechanical ventilation invasive or non-invasive). Exclusion criteria: known hypersensitivity to tocilizumab, coexisting infection that may have worsened, more than 24 hours have passed since ICU admission or more than 24 hours after starting respiratory support, a baseline alanine aminotransferase (ALT) or aspartate aminotransferase (AST) more than 5 times the upper limit of normal, a platelet count below $50 \times 109$ / L, absolute neutrophil count at baseline less than $2 \times 109$ / L, a pre-existing condition or treatment that results in ongoing immunosuppression.

Using AIFA guidelines, consequently, the number of patients who can use TZC is very low; nevertheless, $23,2 \%$ of male used TCZ. Cost of every vial is $€ 211,00$. Total costs were showing in Table 3. 
Table 3. Differences by gender regarding therapeutic measures, as well as imaging and laboratory findings.

\begin{tabular}{|l|l|l|}
\hline Cost & Female $(\mathrm{n}=991)$ & Male $(+295$ than female $)$ \\
\hline Hospitalization & $\begin{array}{l}+1 \text { day, }(€ 546,00) \text { than } \\
\text { female }\end{array}$ \\
\hline TCZ $(€ 211,00 /$ vial $)$ & +271 than female \\
\hline total & $\begin{array}{l}+€ 747871,00(€ 591,2 / \mathrm{man}) \\
\text { than female }\end{array}$ \\
\hline
\end{tabular}

\section{Discussion}

Based on the findings of this study, the differences by gender in epidemiological link, chest $\mathrm{X}$-ray findings, oxygen therapy, comorbidity and treatment were observed among patients with COVID-19. In male subjects, the epidemiological link was related to rehabilitation ward and community, while in female subjects, the epidemiological link was related to Hospital. In terms of clinical manifestations, the common symptoms of these patients were fever, dyspnea and cough, and no differences by gender were observed. In our study, we observed that hypertension and COPD were significantly increased in male patients. According to chest CT scan findings, $86 \%$ of patients had bilateral pneumonia and the remaining 14\% had unilateral pneumonia. Interesting, we report a significantly increase in atypical chest- $X$ ray for female subjects, while Tocilizumab, antiviral drugs and oxygen therapy, were prescribed more in male subjects. No differences by gender we report in other treatment. No strong significantly differences by gender we report in death.

Different reasons can describe the gender differences in relation to COVID-19. It is reported that for both, males and females, lung involvement increased significantly as age increased. It has been described that, males older 50 years, and females older 80 years, with COVID-19 pneumonia, showed the highest Chest X-ray score (13). Chest X-ray COVID-19 reporting classification is based upon recommendation from Radiological society of North America as typical having multifocal peripheral opacities with differential diagnosis of drug toxicity, influenza pneumonia and organizational pneumonia, indeterminate as non-peripheral consolidation with differential of lots of infectious processes, atypical with uncommon imaging features and negative (14).

Atypical chest- $X$ ray in female subjects, compared to male subjects $(41 \%$ vs $16 \%$, respectively, $\mathrm{p}<0.001$ ) and typical chest $\mathrm{X}$-ray, was frequently observed in male compared to female subjects ( $84 \%$ vs $59 \%$; respectively, $\mathrm{p}<0.001$ ).

Aging is another factor that induces the modification of immune response. In fact, with aging, there is an aberrant chronic low-grade pro-inflammatory state, which may occur to a greater extent in females than in males (15). To this regard, immune senescence is a major contributory factor in the increased susceptibility of older adults to infection (14-15). In relation to epidemiological link, for men subjects, it was related to rehabilitation and community, while for female subjects it was related to hospital admission. Probably, according to literature (16), these data are related to hospital contagious. In fact, it has been reported that in Italy, about 11\% of COVID-19 patients were medical or nurse and about $68 \%$ of these are represented by female subjects. It suggests an intrahospital or unnoticed transmission, with a potential risk of diffusion and this role exposed drastically 
the female subjects to contagious. No data in literature describe the role of epidemiological link to contagious in relation to gender.

Because Covid-19 is an emerging infectious disease, the optimal treatment for affected individuals has not yet been established. In our study, most patients were treated with hydroxychloroquine, lopinavir, remdesivir, tocilizumab, Low-molecular-weight heparin (LMWH), corticosteroids, and antibiotics. Although remdesivir and other antiviral drugs have been used in the clinical treatment of patients with COVID-19, few data of their safety and efficacy, as COVID-19 treatments, have been published by gender. To this regard, we report that tocilizumab (TCZ) was prescribed in $14 \%$ of our population. It prescribed in $23 \%$ of male subjects compare to only $2 \%$ of female subjects $(p<0.001)$. We do not know the reasons for these differences. Probably, it is correlated with significantly decrease in adverse reaction in male subjects. Unfortunately, no data described in literature, the gender differences on the TCZ treatment. In fact, there are not data on reallife about the effect by gender of TCZ on the inflammatory activity in COVID-19 patients. Fortunately, the clinical characteristics of these patients with COVID-19 are characterized by very good outcomes in relation to death by gender. In fact, in our study, only $9 \%$ of patients died and no strong significantly differences we reported by gender. Clinical significance of this study is to provide an early insight into atypical chest X-ray finding in female patients that does not exclude COVID-19 infection. Our study confirmed that the evaluation of the tocilizumab (TCZ) effect in COVID-19 patients, by gender, requires controlled studies (ideally RCTs) to evaluate the efficacy and adverse reaction by gender. However, rehabilitation ward, community and hospital can be unnoticed cause of infection in male or female gender.

\section{Limitations}

The retrospective aspect may introduce selection bias and misclassification/information bias. The representativeness of the sample is significant but not representative of the Italian reality because it is limited to a single Region. More infected patients and comparative studies (e.g., cohorts, case-control) should be analyzed to get a more comprehensive understanding of COVID-19 by gender.

\section{Conclusions}

Co-St is one of the few studies that investigates the relationship between covid, gender medicine and costs related to hospitalization and care. men cost more not only because they represent the majority of patients, but also because they require longer and more expensive treatments. This study investigates only the costs related to acute care, and it will be necessary to expand it with direct and indirect costs related to relationship between gender differences and the so-called Long Covid.

Author Contributions: Conceptualization, TC and FP; methodology, SR and AF; software, RC; validation, GC; formal analysis, GS; investigation, EP; resources, TC, FP, AF ; data curation, GC; writing-original draft preparation, TC, MG and GC; writing-review and editing, FR; visualization, OVG; supervision, FL. All authors have read and agreed to the published version of the manuscript."

Funding: This research received no external funding

Institutional Review Board Statement: The study was conducted according to the guidelines of the Declaration of Helsinki and approved by the Ethics Committee (protocol code "55.21 Study", protocol 78111 04/05/2021).

Informed consent was obtained from all subjects involved in the study.

Data Availability Statement: The data presented in this study are available on request from the corresponding author. The data are not publicly available due to patient's privacy. 
Acknowledgments: We thank the doctor Cecilia Politi, Internal Medicine Department Hospital F. Veneziale Isernia (Italy), Responsabile Area Medicina di Genere FADOI.

Conflicts of Interest: The authors declare no conflict of interest.

\section{References}

1. Perlman S. Another Decade, Another Coronavirus. N Engl J Med 2020; 382:760.

2. WHO Director-General's opening remarks at the media briefing on COVID-19 - 11 February 2020.

3. John Hopkins University \& Medicine, Coronavirus Resource Center.

4. Grasselli G, Zangrillo A, Zanella A, et al, COVID-19. Lombardy ICU Network. Baseline characteristics and outcomes of 1591 patients infected with SARS-CoV-2 admitted to ICUs of the Lombardy region, Italy. JAMA 2020; doi: 10.1001/jama.2020.5394.

5. Ilardi AA, Politi C, Ciarambino T. COVID-19: could sex and age be a risk factor? Minerva Med. 2020 Jul 20.doi: 10.23736/S00264806.20.06705-1.

6. Astha Tejpal, Eugenia Gianos, Jane Cerise, et al. Sex-Based Differences in COVID-19 Outcomes. Journal of Women's Health. Apr 2021.492-501.

7. Tisha Suboc, Joanne Michelle D. Gomez, et al. Lessons Learned from Coronavirus Disease 2019 Sex Disparities. Journal of Women's Health. Apr 2021.453-454.

8. Fink AL, Klein SL. Sex and Gender Impact Immune Responses to Vaccines Among the Elderly. Physiology (Bethesda). 2015;30(6):408-416.

9. Parikh P, Wicher S, Khandalavala K, et al. Cellular senescence in the lung across the age spectrum. Am J Physiol Lung Cell Mol Physiol. (2019) 316: L826-L842. doi: 10.1152/ajplung.004 24.2018.

10. Huang C, Wang Y, Li X, et al. Clinical features of patients infected with 2019 novel coronavirus in Wuhan, China. Lancet 2020; 395: 497-506.

11. Borghesi A., Zigliani A., Maroldi R. Radiographic severity index in Covid-19 pneumonia: relationship to age and sex in 783 Italian patients. Radiologica Medica 2020.

12. F. Pietrantonio, L. Piasini, F. Spandonaro. Internal Medicine and emergency admissions: from a national Hospital Discharge Records (SDO) study to a regional analysis. Italian Journal of Medicine Italian Journal of Medicine 2016; volume 10:157-167

13. Simpson S, Kay FU, Abbara S, et al. Radiological Society of North America Expert Consensus Statement on Reporting Chest CT Findings Related to COVID-19. Endorsed by the Society of Thoracic Radiology, the American College of Radiology, and RSNA. Radiol Cardiothorac Imaging. 2020;2(2): e200152.

14. Fink AL, Klein SL. Sex and Gender Impact Immune Responses to Vaccines Among the Elderly. Physiology (Bethesda). 2015;30(6):408-416.

15. Molony RD, Malawista A, Montgomery RR. Reduced dynamic range of antiviral innate immune responses in aging. Exp Gerontol. (2018) 107:130- 5. doi: 10.1016/j.exger.2017.08.019. 